\title{
Synthesis, Characterization and In Vitro Cytotoxicity of Platinum (II) Complexes with Some Tetradentate Salen Ligands
}

\author{
Quang Trung Nguyen*, Quang Hai Lam, Phuong Nam Pham Thi, Van Tuyen Nguyen \\ Institute of Chemistry, Vietnam Academy of Science and Technology, Hanoi, Vietnam \\ Email address: \\ trungquang_cnhh@yahoo.com (Q. T. Nguyen) \\ ${ }^{*}$ Corresponding author

\section{To cite this article:} \\ Quang Trung Nguyen, Quang Hai Lam, Phuong Nam Pham Thi, Van Tuyen Nguyen. Synthesis, Characterization and In Vitro Cytotoxicity of \\ Platinum (II) Complexes with Some Tetradentate Salen Ligands. Science Journal of Chemistry. Vol. 7, No. 2, 2019, pp. 49-55. \\ doi: $10.11648 /$ j.sjc. 20190702.13
}

Received: May 15, 2019; Accepted: June 17, 2019; Published: July 1, 2019

\begin{abstract}
A series of platinum (II) complexes of Schiff bases derived from various salicylaldehydes with ethylenediamine were synthesized and characterized by ESI-MS, NMR and IR. The results of ESI-MS and NMR spectra data showed that the separation of hydrogen from hydoxy group and different chemical shifts of typical bondings between ligands and platinum (II) complexes evidenced for coordination of Pt (II) and tetradentate ligands. The IR spectral studies indicated the binding sites of the salen ligands with Pt (II). Their photophysical properties studied by UV-vis and luminescent spectroscopies performed the photoactivity of investigated platinum (II) complexes. The obvious effect of electron-withdrawing and - donoring substituted groups at the same position of salicyl rings and the impact of methoxy at different position of salicyl rings to their photophysical properties were studied and compared to complex 1 without substituted group. The obtained complexes were screened for their in vitro antitumour activities against KB and MCF-7 human cancer cell lines. Complex 4 showed the best bioactivity for both $\mathrm{KB}$ and MCF-7 human cancer cells with $\mathrm{IC}_{50} 1.92 \mu \mathrm{M}$.
\end{abstract}

Keywords: Salicylaldiminato Pt (II) Complexes, Synthesis, Photophysical Properties, Cytotoxicity

\section{Introduction}

The modern application of transition metal complexes as chemotherapeutic agents came from the discovery of cisplatin by Rosenberg et al. in 1965 and this discovery opened a new gate of metal-based chemotherapeutic agents which have different kinetics and mechanism of action from those of conventional organic drugs [1]. Cisplatin, carboplatin and oxaliplatin approved by FDA are platinum anticancer drugs that are used in clinic world-wide for the treatment of various cancers [2]. However, severe side effects, drug resistance and poor targeted delivery are the major problems for wider clinical application of these drugs [3]. Bioinorganic and medicinal chemists are exploring different strategies to overcome these obstacles, which includes targeted delivery of clinical drugs [4] and design of new novel platinum and non-platinum metal complexes which have different structural features and reactivities [5].
Schiff bases are considered as a very important class of organic compounds which have wide applications in many biological aspects [6]. Schiff base ligands can form quite stable complexes with many transition metals, with different oxidation states, coordination numbers and geometries [7]. Schiff base complexes are used as model molecules for biological oxygen carrier systems. Some of these complexes have applications in analytical fields [8]. Recently some tetradentate salicylaldiminato $\mathrm{Pt}$ (II) complexes were synthesized and estimated for their photophysical properties [9-14] and cytotoxicity [15-18]. Because of tetradentate salicylaldiminato Pt (II) complexes' potential in chemotherapy for various human cancer cell lines, in this research we continue to study on Pt (II) complexes of tetradentate salicylaldimine ligands derived from various salicylaldehydes with ethylenediamine and estimate their photophysical properties and in vitro cytotoxicity for $\mathrm{KB}$ and MCF-7 human cancer cell lines as well. 


\section{Experimental and Results}

\subsection{Chemicals and Instrumentation}

All chemicals used in the synthesis were of reagent grade and obtained from commercial companies. Solvents were purified by standard procedures before using. IR spectra at 4000-400 $\mathrm{cm}^{-1}$ were recorded on Perkin-Elmer Spectrum Two. ${ }^{1} \mathrm{H}$ and ${ }^{13} \mathrm{C}$-NMR were obtained on Bruker Avance 500 at 500 and $150 \mathrm{MHz}$ with TMS as internal standard and chemical shifts $(\delta)$ were reported in ppm. ESI-MS was determined on Agilent 6310 Ion Trap in analytical center of Vietnam Institute of Chemistry. Reactions were all monitored by TLC, performed on silica gel plates 60 F254 (Merck, Germany). Visualization on TLC was achieved by UV lamb.

\subsection{Synthesis of Salen Ligands}

Generally salen ligands were synthesized following the published paper [19] by mixing two equivalents of salicylaldehyde (or derivatives) with one equivalent of ethylenediamine in ethanol at room temperature that resulted in yellow colored precipitate of respective salen derivatives. The precipitate was filtered, washed with cold ethanol and dried under reduced pressure. Each of the ligand was characterized by ESI-MS, IR, ${ }^{1} \mathrm{H}$ and ${ }^{13} \mathrm{C}$ NMR spectra and the results are consistent with their structures.

$N, N^{\prime}$-Bis (salicylidene) ethylene diimine $\left(\right.$ salen $\left.\mathrm{H}_{2}\right)$

Yellow solid product, yield 93\%. ESI-MS (m/z) 268.9 $[\mathrm{M}+\mathrm{H}]^{+}$. IR $\left(\mathrm{KBr}, \mathrm{cm}^{-1}\right): 2900(v, \mathrm{C}-\mathrm{H}) ; 2549(v, \mathrm{O}-\mathrm{H})$; $1636(v, \mathrm{C}=\mathrm{N}) ; 1497(\mathrm{v}, \mathrm{C}=\mathrm{C}) ; 1283(\mathrm{v}, \mathrm{C}-\mathrm{N}) ; 1199(\mathrm{v}, \mathrm{C}-$ $\mathrm{O}) ; 1042 ; 856 ; 742(\delta, \mathrm{C}-\mathrm{H}) .{ }^{1} \mathrm{H}-\mathrm{NMR}\left(\mathrm{CDCl}_{3}, \mathrm{ppm}, \mathrm{Hz}\right): \delta$ $13.18(\mathrm{~s}, 2 \mathrm{H}, 2 \mathrm{OH}) ; 8.35(\mathrm{~s}, 2 \mathrm{H}, 2 \mathrm{CH}=\mathrm{N}) ; 7.28(\mathrm{dt}, J=8.0$; 2.0, 2H, Ar-H); $7.21(\mathrm{dd}, J=7.5 ; 2.0,2 \mathrm{H}, \mathrm{Ar}-\mathrm{H}) ; 6.93$ (d, $J$ $=8.0,2 \mathrm{H}, \mathrm{Ar}-\mathrm{H}) ; 6.84(\mathrm{dt}, J=7.5 ; 1.0,2 \mathrm{H}, \mathrm{Ar}-\mathrm{H}) ; 3.93(\mathrm{~s}$, $\left.4 \mathrm{H}, \mathrm{CH}_{2}-\mathrm{CH}_{2}\right) .{ }^{13} \mathrm{C}-\mathrm{NMR}\left(\mathrm{CDCl}_{3}, \mathrm{ppm}\right): \delta 166.51(2 \mathrm{C}$, $2 \mathrm{C}-\mathrm{O}) ; 161.03(2 \mathrm{C}, 2 \mathrm{C}=\mathrm{N}) ; 132.40\left(2 \mathrm{C}, \mathrm{C}_{\mathrm{Ar}}\right) ; 131.49(2 \mathrm{C}$, $\left.\mathrm{C}_{\mathrm{Ar}}\right) ; 118.69\left(2 \mathrm{C}, \mathrm{C}_{\mathrm{Ar}}\right) ; 118.67\left(2 \mathrm{C}, \mathrm{C}_{\mathrm{Ar}}\right) ; 116.97\left(2 \mathrm{C}, \mathrm{C}_{\mathrm{Ar}}\right)$; $59.75\left(2 \mathrm{C}, \mathrm{CH}_{2}-\mathrm{CH}_{2}\right)$.

$N$, N'-Bis (5-fluorosalicylidene) ethylene diimine (5FsalenH $\mathrm{H}_{2}$ )

Yellow solid product, yield 95\%. ESI-MS (m/z) 304.9 $[\mathrm{M}+\mathrm{H}]^{+}$. IR $\left(\mathrm{KBr}, \mathrm{cm}^{-1}\right): 2911(v, \mathrm{C}-\mathrm{H}) ; 2571(v, \mathrm{O}-\mathrm{H})$; $1634(v, \mathrm{C}=\mathrm{N}) ; 1498(v, \mathrm{C}=\mathrm{C}) ; 1364 ; 1272(v, \mathrm{C}-\mathrm{N}) ; 1225$ (v, C-O); 1041; 828; $782(\delta, \mathrm{C}-\mathrm{H}) .{ }^{1} \mathrm{H}-\mathrm{NMR}\left(\mathrm{CDCl}_{3}, \mathrm{ppm}\right)$ : $\delta 12.85(\mathrm{~s}, 2 \mathrm{H}, 2 \mathrm{OH}) ; 8.30(\mathrm{~s}, 2 \mathrm{H}, 2 \mathrm{CH}=\mathrm{N}) ; 7.02(\mathrm{~m}, 2 \mathrm{H}$, $\mathrm{Ar}-\mathrm{H}) ; 6.93$ (dd, $J=8.5 ; 3.0,2 \mathrm{H}, \mathrm{Ar}-\mathrm{H}) ; 6.89$ (dd, $J=8.0$; 4.0, $2 \mathrm{H}, \mathrm{Ar}-\mathrm{H}) ; 3.96\left(\mathrm{~s}, 4 \mathrm{H}, \mathrm{CH}_{2}-\mathrm{CH}_{2}\right) .{ }^{13} \mathrm{C}-\mathrm{NMR}\left(\mathrm{CDCl}_{3}\right.$, ppm): $\delta 165.58$ and $165.56(2 \mathrm{C}, 2 \mathrm{C}-\mathrm{O}) ; 154.60(2 \mathrm{C}, 2 \mathrm{C}=\mathrm{N})$; 157.25 and $156.48(2 \mathrm{C}, 2 \mathrm{C}-\mathrm{F}) ; 119.65$ and $119.47\left(2 \mathrm{C}, \mathrm{C}_{\mathrm{Ar}}\right)$; 118.48 and $118.43\left(2 \mathrm{C}, \mathrm{C}_{\mathrm{Ar}}\right) ; 118.17$ and $118.11\left(2 \mathrm{C}, \mathrm{C}_{\mathrm{Ar}}\right)$; 116.61 and $116.43\left(2 \mathrm{C}, \mathrm{C}_{\mathrm{Ar}}\right) ; 59.71\left(2 \mathrm{C}, \mathrm{CH}_{2}-\mathrm{CH}_{2}\right)$.

$N$, N'-Bis (3-methoxysalicylidene) ethylene diimine (3MeOsalenH $\mathrm{H}_{2}$ )

Yellow solid product, yield 91\%. ESI-MS (m/z) 329.0 $[\mathrm{M}+\mathrm{H}]^{+} . \mathrm{IR}\left(\mathrm{KBr}, \mathrm{cm}^{-1}\right): 2921(v, \mathrm{C}-\mathrm{H}) ; 2555(v, \mathrm{O}-\mathrm{H})$; $1633(v, C=N) ; 1470(v, C=C) ; 1296(v, C-N) ; 1250(v, C-$ O); 1081; 963; $741(\delta, \mathrm{C}-\mathrm{H}) .{ }^{1} \mathrm{H}-\mathrm{NMR}\left(\mathrm{CDCl}_{3}, \mathrm{ppm}\right): \delta$ $13.55(\mathrm{~s}, 2 \mathrm{H}, 2 \mathrm{OH}) ; 8.32(\mathrm{~s}, 2 \mathrm{H}, 2 \mathrm{CH}=\mathrm{N}) ; 6.91(\mathrm{dd}, J=8.0$; $1.5,2 \mathrm{H}, \mathrm{Ar}-\mathrm{H}) ; 6.85(\mathrm{dd}, J=8.0 ; 1.5,2 \mathrm{H}, \mathrm{Ar}-\mathrm{H}) ; 6.77(\mathrm{t}, J=$ 8.0, $2 \mathrm{H}, \mathrm{Ar}-\mathrm{H}) ; 3.95\left(\mathrm{~s}, 4 \mathrm{H}, \mathrm{CH}_{2}-\mathrm{CH}_{2}\right) ; 3.88(\mathrm{~s}, 6 \mathrm{H}, 2 \mathrm{MeO})$. ${ }^{13} \mathrm{C}-\mathrm{NMR}\left(\mathrm{CDCl}_{3}, \mathrm{ppm}\right): \delta 166.68(2 \mathrm{C}, 2 \mathrm{C}-\mathrm{O}) ; 151.49(2 \mathrm{C}$, $2 \mathrm{C}=\mathrm{N}) ; 148.34(2 \mathrm{C}, 2 \mathrm{C}-\mathrm{OMe}) ; 123.21\left(2 \mathrm{C}, \mathrm{C}_{\mathrm{Ar}}\right) ; 118.49$ $\left(2 \mathrm{C}, \mathrm{C}_{\mathrm{Ar}}\right) ; 118.06\left(2 \mathrm{C}, \mathrm{C}_{\mathrm{Ar}}\right) ; 114.23\left(2 \mathrm{C}, \mathrm{C}_{\mathrm{Ar}}\right) ; 59.50(2 \mathrm{C}$, $\left.\mathrm{CH}_{2}-\mathrm{CH}_{2}\right) ; 56.11(2 \mathrm{C}, 2 \mathrm{MeO})$.

$N$, N'-Bis (4-methoxysalicylidene) ethylene diimine (4MeOsalenH $\mathrm{H}_{2}$ )

Yellow solid product, yield 91\%. ESI-MS (m/z) 329.0 $[\mathrm{M}+\mathrm{H}]^{+}$. IR $\left(\mathrm{KBr}, \mathrm{cm}^{-1}\right): 2927(v, \mathrm{C}-\mathrm{H}) ; 2551(v, \mathrm{O}-\mathrm{H})$; $1620(v, \mathrm{C}=\mathrm{N}) ; 1512(v, \mathrm{C}=\mathrm{C}) ; 1285(v, \mathrm{C}-\mathrm{N}) ; 1223(\mathrm{v}, \mathrm{C}-$ O); $1114 ; 964 ; 852,800(\delta, \mathrm{C}-\mathrm{H}) .{ }^{1} \mathrm{H}-\mathrm{NMR}\left(\mathrm{CDCl}_{3}, \mathrm{ppm}\right): \delta$ $13.68(\mathrm{~s}, 2 \mathrm{H}, 2 \mathrm{OH}) ; 8.20(\mathrm{~s}, 2 \mathrm{H}, 2 \mathrm{CH}=\mathrm{N}) ; 7.08(\mathrm{~d}, J=8.5$, $2 \mathrm{H}, \mathrm{Ar}-\mathrm{H}) ; 6.41$ (d, $J=2.5,2 \mathrm{H}, \mathrm{Ar}-\mathrm{H}) ; 6.37$ (dd, $J=8.5$; 2.5, $2 \mathrm{H}, \mathrm{Ar}-\mathrm{H}) ; 3.85\left(\mathrm{~s}, 4 \mathrm{H}, \mathrm{CH}_{2}-\mathrm{CH}_{2}\right) ; 3.79$ (s, 6H, 2MeO). ${ }^{13} \mathrm{C}-\mathrm{NMR}\left(\mathrm{CDCl}_{3}, \mathrm{ppm}\right): \delta 165.43(2 \mathrm{C}, 2 \mathrm{C}-\mathrm{O}) ; 164.65(2 \mathrm{C}$, $2 \mathrm{C}-\mathrm{OMe}) ; 163.53(2 \mathrm{C}, 2 \mathrm{C}=\mathrm{N}) ; 132.71\left(2 \mathrm{C}, \mathrm{C}_{\mathrm{Ar}}\right) ; 112.36$ $\left(2 \mathrm{C}, \mathrm{C}_{\mathrm{Ar}}\right) ; 106.43\left(2 \mathrm{C}, \mathrm{C}_{\mathrm{Ar}}\right) ; 101.16\left(2 \mathrm{C}, \mathrm{C}_{\mathrm{Ar}}\right) ; 58.85(2 \mathrm{C}$, $\left.\mathrm{CH}_{2}-\mathrm{CH}_{2}\right) ; 55.34(2 \mathrm{C}, 2 \mathrm{MeO})$.

$N$, N'-Bis (5-methoxysalicylidene) ethylene diimine (5MeOsalenH $\mathrm{H}_{2}$ )

Yellow solid product, yield 92\%. ESI-MS (m/z) 329.0 $[\mathrm{M}+\mathrm{H}]^{+}$. IR $\left(\mathrm{KBr}, \mathrm{cm}^{-1}\right): 2937(v, \mathrm{C}-\mathrm{H}) ; 2572(v, \mathrm{O}-\mathrm{H})$; $1639(v, \mathrm{C}=\mathrm{N}) ; 1492(v, \mathrm{C}=\mathrm{C}) ; 1276(v, \mathrm{C}-\mathrm{N}) ; 1159(v, \mathrm{C}-$ $\mathrm{O}) ; 1031 ; 829 ; 784(\delta, \mathrm{C}-\mathrm{H}) .{ }^{1} \mathrm{H}-\mathrm{NMR}\left(\mathrm{CDCl}_{3}, \mathrm{ppm}\right): \delta$ $12.68(\mathrm{~s}, 2 \mathrm{H}, 2 \mathrm{OH}) ; 8.30(\mathrm{~s}, 2 \mathrm{H}, 2 \mathrm{CH}=\mathrm{N}) ; 6.90(\mathrm{dd}, J=9.0$; 3.0, 2H, Ar-H); 6.87 (d, $J=9.0,2 \mathrm{H}, \mathrm{Ar}-\mathrm{H}) ; 6.73$ (d, $J=3.0$, $2 \mathrm{H}, \mathrm{Ar}-\mathrm{H}) ; 3.94\left(\mathrm{~s}, 4 \mathrm{H}, \mathrm{CH}_{2}-\mathrm{CH}_{2}\right) ; 3.75(\mathrm{~s}, 6 \mathrm{H}, 2 \mathrm{MeO}) .{ }^{13} \mathrm{C}-$ NMR $\left(\mathrm{CDCl}_{3}, \mathrm{ppm}\right): \delta 166.27(2 \mathrm{C}, 2 \mathrm{C}-\mathrm{O}) ; 155.17(2 \mathrm{C}$, $2 \mathrm{C}=\mathrm{N}) ; 152.06(2 \mathrm{C}, 2 \mathrm{C}-\mathrm{OMe}) ; 119.56\left(2 \mathrm{C}, \mathrm{C}_{\mathrm{Ar}}\right) ; 118.27$ $\left(2 \mathrm{C}, \mathrm{C}_{\mathrm{Ar}}\right) ; 117.70\left(2 \mathrm{C}, \mathrm{C}_{\mathrm{Ar}}\right) ; 114.98\left(2 \mathrm{C}, \mathrm{C}_{\mathrm{Ar}}\right) ; 59.85(2 \mathrm{C}$, $\left.\mathrm{CH}_{2}-\mathrm{CH}_{2}\right) ; 55.95(2 \mathrm{C}, 2 \mathrm{MeO})$.

$N$, N'-Bis (6-methoxysalicylidene) ethylene diimine (6$\mathrm{MeOsalenH}_{2}$ )

Yellow solid product, yield 91\%. ESI-MS (m/z) 329.0 $[\mathrm{M}+\mathrm{H}]^{+} . \mathrm{IR}\left(\mathrm{KBr}, \mathrm{cm}^{-1}\right): 2937(v, \mathrm{C}-\mathrm{H}) ; 2532(v, \mathrm{O}-\mathrm{H})$; $1627(v, \mathrm{C}=\mathrm{N}) ; 1462(v, \mathrm{C}=\mathrm{C}) ; 1295(v, \mathrm{C}-\mathrm{N}) ; 1250(v, \mathrm{C}-$ $\mathrm{O}) ; 1099 ; 837 ; 780(\delta, \mathrm{C}-\mathrm{H}) ; 722 .{ }^{1} \mathrm{H}-\mathrm{NMR}\left(\mathrm{CDCl}_{3}, \mathrm{ppm}\right): \delta$ $14.18(\mathrm{~s}, 2 \mathrm{H}, 2 \mathrm{OH}) ; 8.80(\mathrm{~s}, 2 \mathrm{H}, 2 \mathrm{CH}=\mathrm{N}) ; 7.19(\mathrm{t}, J=8.5$, $2 \mathrm{H}, \mathrm{Ar}-\mathrm{H}) ; 6.51(\mathrm{~d}, J=8.5,2 \mathrm{H}, \mathrm{Ar}-\mathrm{H}) ; 6.25(\mathrm{~d}, J=8.5,2 \mathrm{H}$, $\mathrm{Ar}-\mathrm{H}) ; 3.88\left(\mathrm{~s}, 4 \mathrm{H}, \mathrm{CH}_{2}-\mathrm{CH}_{2}\right) ; 3.78(\mathrm{~s}, 6 \mathrm{H}, 2 \mathrm{MeO}) .{ }^{13} \mathrm{C}-$ NMR $\left(\mathrm{CDCl}_{3}, \mathrm{ppm}\right): \delta 163.80(2 \mathrm{C}, 2 \mathrm{C}-\mathrm{O}) ; 162.63(2 \mathrm{C}$, $2 \mathrm{C}=\mathrm{N}) ; 159.64(2 \mathrm{C}, 2 \mathrm{C}-\mathrm{OMe}) ; 133.46\left(2 \mathrm{C}, \mathrm{C}_{\mathrm{Ar}}\right) ; 110.24$ $\left(2 \mathrm{C}, \mathrm{C}_{\mathrm{Ar}}\right) ; 108.08\left(2 \mathrm{C}, \mathrm{C}_{\mathrm{Ar}}\right) ; 99.78\left(2 \mathrm{C}, \mathrm{C}_{\mathrm{Ar}}\right) ; 59.65(2 \mathrm{C}$, $\left.\mathrm{CH}_{2}-\mathrm{CH}_{2}\right) ; 55.57$ (2C, 2MeO).

\subsection{Synthesis of Salen Pt (II) Complexes}

Pt (II)-salen derivatives were synthesized and characterized following a general procedure as described previously [20]. In general, for the synthesis of Pt (II)-salen derivatives, the respective salen ligand derivatives were dissolved DMSO and then mixed with equivalent amount of $\mathrm{K}_{2} \mathrm{PtCl}_{4}$ (200 mg, $\left.0.48 \mathrm{mmol}\right)$ in DMSO. Then aqueous saturated solution of $\mathrm{Na}_{2} \mathrm{CO}_{3}(52 \mathrm{mg}, 0.48 \mathrm{mmol})$ was added. The reaction mixture was stired continuously and refluxed for $3 \mathrm{~h}$. After cooling to room temperature the yellow 
precipitate was filtered and washed by distilled water and cold ethenol, then dried in reduced vacuo to afford a yellow solid product. The products were isolated, recrystallized from ethyl ether and characterized by mass spectral (ESI-MS), ${ }^{1} \mathrm{H}$ and ${ }^{13} \mathrm{C}$-NMR, and IR spectra.

[Pt (II) (salen)] (1): Yellow solid, yield 67.5\%. ESI-MS $(\mathrm{m} / \mathrm{z}) 462.1[\mathrm{M}+\mathrm{H}]^{+}$. IR $\left(\mathrm{KBr}, \mathrm{cm}^{-1}\right): 3022(\mathrm{v}, \mathrm{C}-\mathrm{H}) ; 1621(\mathrm{v}$, $\mathrm{C}=\mathrm{N}) ; 1530(v, \mathrm{C}=\mathrm{C}) ; 1439 ; 1309 ; 1192(v, \mathrm{C}-\mathrm{N}) ; 1127(v$, $\mathrm{C}-\mathrm{O}) ; 1081 ; 846 ; 742(\delta, \mathrm{C}-\mathrm{H}) ; 611(\mathrm{Pt}-\mathrm{O}) ; 417(\mathrm{Pt}-\mathrm{N}) .{ }^{1} \mathrm{H}-$ NMR (DMSO- $\left.d_{6}, \mathrm{ppm}\right): 8.54(\mathrm{~s}, 2 \mathrm{H}, 2 \mathrm{CH}=\mathrm{N}) ; 7.50(\mathrm{~d}, J=$ $7.5 \mathrm{~Hz}, 2 \mathrm{H}, \mathrm{Ar}-\mathrm{H}) ; 7.43(\mathrm{t}, J=7.0,2 \mathrm{H}, \mathrm{Ar}-\mathrm{H}) ; 6.91(\mathrm{~d}, J=$ $8.5,2 \mathrm{H}, \mathrm{Ar}-\mathrm{H}) ; 6.61(\mathrm{t}, J=7.0,2 \mathrm{H}, \mathrm{Ar}-\mathrm{H}) ; 3.82(\mathrm{~s}, 4 \mathrm{H}$, $\left.\mathrm{CH}_{2}-\mathrm{CH}_{2}\right) .{ }^{13} \mathrm{C}-\mathrm{NMR}$ (DMSO- $\left.d_{6}, \mathrm{ppm}\right): \delta 162.45(2 \mathrm{C}$, $2 \mathrm{C}-\mathrm{O}) ; 155.98(2 \mathrm{C}, 2 \mathrm{C}=\mathrm{N}) ; 133.70\left(2 \mathrm{C}, \mathrm{C}_{\mathrm{Ar}}\right) ; 133.48(2 \mathrm{C}$, $\left.\mathrm{C}_{\mathrm{Ar}}\right) ; 122.28\left(2 \mathrm{C}, \mathrm{C}_{\mathrm{Ar}}\right) ; 120.94\left(2 \mathrm{C}, \mathrm{C}_{\mathrm{Ar}}\right) ; 115.48\left(2 \mathrm{C}, \mathrm{C}_{\mathrm{Ar}}\right)$; $60.92\left(2 \mathrm{C}, \mathrm{CH}_{2}-\mathrm{CH}_{2}\right)$.

[Pt (II) (5-Fsalen)] (2): Yellow solid, yield 68.5\%. ESI-MS $(\mathrm{m} / \mathrm{z}) 497.9[\mathrm{M}+\mathrm{H}]^{+} . \mathrm{IR}\left(\mathrm{KBr}, \mathrm{cm}^{-1}\right): 3019(\mathrm{v}, \mathrm{C}-\mathrm{H}) ; 1612(\mathrm{v}$, $\mathrm{C}=\mathrm{N}) ; 1545(v, \mathrm{C}=\mathrm{C}) ; 1472 ; 1313 ; 1221(v, \mathrm{C}-\mathrm{N}) ; 1127(v$, $\mathrm{C}-\mathrm{O}) ; 1021 ; 971 ; 807(\delta, \mathrm{C}-\mathrm{H}) ; 618(\mathrm{Pt}-\mathrm{O}) ; 434(\mathrm{Pt}-\mathrm{N}) .{ }^{1} \mathrm{H}-$ NMR (DMSO- $\left.d_{6}, \mathrm{ppm}\right): 8.22(\mathrm{~s}, 2 \mathrm{H}, 2 \mathrm{CH}=\mathrm{N}) ; 7.27(\mathrm{~m}, 2 \mathrm{H}$, Ar-H); 7.18 (dd, $J=9.0 ; 3.5 \mathrm{~Hz}, 2 \mathrm{H}, \mathrm{Ar}-\mathrm{H}) ; 6.91$ (dd, $J=$ $9.5 ; 4.5,2 \mathrm{H}, \mathrm{Ar}-\mathrm{H}) ; 4.46\left(\mathrm{~s}, 4 \mathrm{H}, \mathrm{CH}_{2}-\mathrm{CH}_{2}\right) .{ }^{13} \mathrm{C}-\mathrm{NMR}$ (DMSO- $\left.d_{6}, \mathrm{ppm}\right): \delta 162.62(2 \mathrm{C}, 2 \mathrm{C}-\mathrm{O}) ; 157.98(2 \mathrm{C}, 2 \mathrm{C}=\mathrm{N})$; 153.83 and $151.98(2 \mathrm{C}, 2 \mathrm{C}-\mathrm{F}) ; 123.25$ and $123.06\left(2 \mathrm{C}, \mathrm{C}_{\mathrm{Ar}}\right)$; 120.24 and $120.18\left(2 \mathrm{C}, \mathrm{C}_{\mathrm{Ar}}\right) ; 118.43$ and $118.36\left(2 \mathrm{C}, \mathrm{C}_{\mathrm{Ar}}\right)$; 117.18 and $117.00\left(2 \mathrm{C}, \mathrm{C}_{\mathrm{Ar}}\right) ; 60.49\left(2 \mathrm{C}, \mathrm{CH}_{2}-\mathrm{CH}_{2}\right)$.

[Pt (II) (3-MeOsalen)] (3): Yellow solid, yield 66.0\%. ESIMS (m/z) $522.0[\mathrm{M}+\mathrm{H}]^{+}$. IR $\left(\mathrm{KBr}, \mathrm{cm}^{-1}\right): 3011(\mathrm{v}, \mathrm{C}-\mathrm{H})$; $1604(v, \mathrm{C}=\mathrm{N}) ; 1548(v, \mathrm{C}=\mathrm{C}) ; 1466 ; 1325 ; 1247(v, \mathrm{C}-\mathrm{N})$; 1126 (v, C-O); 1031; 977; $733(\delta, \mathrm{C}-\mathrm{H}) ; 631(\mathrm{Pt}-\mathrm{O}) ; 433$ $(\mathrm{Pt}-\mathrm{N}) .{ }^{1} \mathrm{H}-\mathrm{NMR}\left(\mathrm{DMSO}-d_{6}, \mathrm{ppm}\right): 8.52(\mathrm{~s}, 2 \mathrm{H}, 2 \mathrm{CH}=\mathrm{N})$; 7.08 (dd, $J=8.0 ; 1.5,2 \mathrm{H}, \mathrm{Ar}-\mathrm{H}) ; 7.04$ (dd, $J=8.0 ; 1.5,2 \mathrm{H}$, $\mathrm{Ar}-\mathrm{H}) ; 6.55$ (t, $J=8.0,2 \mathrm{H}, \mathrm{Ar}-\mathrm{H}) ; 3.81\left(\mathrm{~s}, 4 \mathrm{H}, \mathrm{CH}_{2}-\mathrm{CH}_{2}\right)$; $3.77(\mathrm{~s}, 6 \mathrm{H}, 2 \mathrm{MeO}) .{ }^{13} \mathrm{C}-\mathrm{NMR}$ (DMSO- $\left.d_{6}, \mathrm{ppm}\right): \delta 156.00$ $(2 \mathrm{C}, 2 \mathrm{C}-\mathrm{O}) ; 153.53(2 \mathrm{C}, 2 \mathrm{C}=\mathrm{N}) ; 151.32(2 \mathrm{C}, 2 \mathrm{C}-\mathrm{OMe})$; $124.89\left(2 \mathrm{C}, \mathrm{C}_{\mathrm{Ar}}\right) ; 121.83\left(2 \mathrm{C}, \mathrm{C}_{\mathrm{Ar}}\right) ; 114.51\left(2 \mathrm{C}, \mathrm{C}_{\mathrm{Ar}}\right) ; 113.33$ $\left(2 \mathrm{C}, \mathrm{C}_{\mathrm{Ar}}\right) ; 60.92\left(2 \mathrm{C}, \mathrm{CH}_{2}-\mathrm{CH}_{2}\right) ; 55.18(2 \mathrm{C}, 2 \mathrm{MeO})$.

[Pt (II) (4-MeOsalen)] (4): Light yellow solid, yield 65.5\%. ESI-MS (m/z) 522.0 [M+H] $]^{+}$IR $\left(\mathrm{KBr}, \mathrm{cm}^{-1}\right): 3020$ $(v, \mathrm{C}-\mathrm{H}) ; 1600(v, \mathrm{C}=\mathrm{N}) ; 1533(v, \mathrm{C}=\mathrm{C}) ; 1456 ; 1317 ; 1228$ $(v, \mathrm{C}-\mathrm{N}) ; 1124(v, \mathrm{C}-\mathrm{O}) ; 1025 ; 980 ; 828 ;(\delta, \mathrm{C}-\mathrm{H}) ; 625$ $(\mathrm{Pt}-\mathrm{O}) ; 432(\mathrm{Pt}-\mathrm{N}) .{ }^{1} \mathrm{H}-\mathrm{NMR}$ (DMSO- $\left.d_{6}, \mathrm{ppm}\right): 8.02$ (s, 2H, $2 \mathrm{CH}=\mathrm{N}) ; 7.22(\mathrm{~d}, J=9.0,2 \mathrm{H}, \mathrm{Ar}-\mathrm{H}) ; 6.44(\mathrm{~d}, J=2.5,2 \mathrm{H}$, $\mathrm{Ar}-\mathrm{H}) ; 6.25(\mathrm{dd}, J=9.0 ; 2.0,2 \mathrm{H}, \mathrm{Ar}-\mathrm{H}) ; 4.32\left(\mathrm{~s}, 4 \mathrm{H}, \mathrm{CH}_{2}-\right.$ $\mathrm{CH}_{2}$ ); 3.73 (s, 6H, $2 \mathrm{MeO}$ ). ${ }^{13} \mathrm{C}-\mathrm{NMR}$ (DMSO- $\left.d_{6}, \mathrm{ppm}\right): \delta$ $165.41(2 \mathrm{C}, 2 \mathrm{C}-\mathrm{OMe}) ; 163.13(2 \mathrm{C}, 2 \mathrm{C}-\mathrm{O}) ; 161.81$ (2C, $2 \mathrm{C}=\mathrm{N}) ; 136.03\left(2 \mathrm{C}, \mathrm{C}_{\mathrm{Ar}}\right) ; 113.48\left(2 \mathrm{C}, \mathrm{C}_{\mathrm{Ar}}\right) ; 107.37\left(2 \mathrm{C}, \mathrm{C}_{\mathrm{Ar}}\right)$; $100.75\left(2 \mathrm{C}, \mathrm{C}_{\mathrm{Ar}}\right) ; 60.53\left(2 \mathrm{C}, \mathrm{CH}_{2}-\mathrm{CH}_{2}\right) ; 55.39(2 \mathrm{C}, 2 \mathrm{MeO})$.

[Pt (II) (5-MeOsalen)] (5): Yellow solid, yield 67.5\%. ESIMS (m/z) $522.0[\mathrm{M}+\mathrm{H}]^{+}$. IR $\left(\mathrm{KBr}, \mathrm{cm}^{-1}\right): 3002(\mathrm{v}, \mathrm{C}-\mathrm{H})$; $1607(v, C=N) ; 1539(v, C=C) ; 1474 ; 1299 ; 1220(v, C-N)$; 1148 (v, C-O); 1033; 928; $829(\delta, \mathrm{C}-\mathrm{H}) ; 613(\mathrm{Pt}-\mathrm{O}) ; 427$ $(\mathrm{Pt}-\mathrm{N}) .{ }^{1} \mathrm{H}-\mathrm{NMR}\left(\mathrm{DMSO}-d_{6}, \mathrm{ppm}\right): 8.47(\mathrm{~s}, 2 \mathrm{H}, 2 \mathrm{CH}=\mathrm{N})$; $7.14(\mathrm{dd}, J=9.5 ; 3.5,2 \mathrm{H}, \mathrm{Ar}-\mathrm{H}) ; 7.00(\mathrm{~d}, J=3.0,2 \mathrm{H}, \mathrm{Ar}-$ $\mathrm{H}) ; 6.84(\mathrm{~d}, J=9.5,2 \mathrm{H}, \mathrm{Ar}-\mathrm{H}) ; 3.80\left(\mathrm{~s}, 4 \mathrm{H}, \mathrm{CH}_{2}-\mathrm{CH}_{2}\right) ; 3.69$ (s, 6H, $2 \mathrm{MeO}) .{ }^{13} \mathrm{C}-\mathrm{NMR}$ (DMSO- $\left.d_{6}, \mathrm{ppm}\right): \delta 157.73(2 \mathrm{C}$, $2 \mathrm{C}-\mathrm{O}) ; 155.41(2 \mathrm{C}, 2 \mathrm{C}=\mathrm{N}) ; 149.23(2 \mathrm{C}, 2 \mathrm{C}-\mathrm{OMe}) ; 123.22$ $\left(2 \mathrm{C}, \mathrm{C}_{\mathrm{Ar}}\right) ; 121.67\left(2 \mathrm{C}, \mathrm{C}_{\mathrm{Ar}}\right) ; 120.85\left(2 \mathrm{C}, \mathrm{C}_{\mathrm{Ar}}\right) ; 113.81(2 \mathrm{C}$, $\left.\mathrm{C}_{\mathrm{Ar}}\right) ; 60.95\left(2 \mathrm{C}, \mathrm{CH}_{2}-\mathrm{CH}_{2}\right) ; 55.53(2 \mathrm{C}, 2 \mathrm{MeO})$.

[Pt (II) (6-MeOsalen)] (6): Yellow solid, yield 67.0\%. ESIMS (m/z) $522.0[\mathrm{M}+\mathrm{H}]^{+}$. IR $\left(\mathrm{KBr}, \mathrm{cm}^{-1}\right): 3006(v, \mathrm{C}-\mathrm{H})$; $1607(v, C=N) ; 1545(v, C=C) ; 1455 ; 1317 ; 1254(v, C-N)$; 1106 (v, C-O); 1040; $778(\delta, \mathrm{C}-\mathrm{H}) ; 623(\mathrm{Pt}-\mathrm{O}) ; 434(\mathrm{Pt}-\mathrm{N})$. ${ }^{1} \mathrm{H}-\mathrm{NMR}$ (DMSO- $\left.d_{6}, \mathrm{ppm}\right): 8.77(\mathrm{~s}, 2 \mathrm{H}, 2 \mathrm{CH}=\mathrm{N}) ; 7.29(\mathrm{t}, J$ $=8.0,2 \mathrm{H}, \mathrm{Ar}-\mathrm{H}) ; 6.51(\mathrm{~d}, J=3.5,2 \mathrm{H}, \mathrm{Ar}-\mathrm{H}) ; 6.20(\mathrm{~d}, J=$ 8.0, $2 \mathrm{H}, \mathrm{Ar}-\mathrm{H}) ; 3.82\left(\mathrm{~s}, 10 \mathrm{H}, \mathrm{CH}_{2}-\mathrm{CH}_{2}\right.$ and $\left.2 \mathrm{MeO}\right) .{ }^{13} \mathrm{C}-$ NMR (DMSO- $\left.d_{6}, \mathrm{ppm}\right): \delta 163.21(2 \mathrm{C}, 2 \mathrm{C}-\mathrm{O}) ; 159.36(2 \mathrm{C}$, $2 \mathrm{C}=\mathrm{N}) ; 149.78(2 \mathrm{C}, 2 \mathrm{C}-\mathrm{OMe}) ; 132.83\left(2 \mathrm{C}, \mathrm{C}_{\mathrm{Ar}}\right) ; 114.25$ $\left(2 \mathrm{C}, \mathrm{C}_{\mathrm{Ar}}\right) ; 112.43\left(2 \mathrm{C}, \mathrm{C}_{\mathrm{Ar}}\right) ; 96.98\left(2 \mathrm{C}, \mathrm{C}_{\mathrm{Ar}}\right) ; 61.58(2 \mathrm{C}$, $\left.\mathrm{CH}_{2}-\mathrm{CH}_{2}\right) ; 55.84(2 \mathrm{C}, 2 \mathrm{MeO})$.

\subsection{Photophysical Properties}

The UV-Visible absorption spectra of investigated platinum (II) complexes were recorded in DCM (dichlorometane) solutions $\left(2 \times 10^{-5} \mathrm{M}\right)$ on Perkin Elmer Lambda UV-35 spectrophotometer. The luminescent spectra of the solutions were measured on Horiba Fluorolog spectrofluorometer at room temperature. The received photophysical data were collected in Table 1.

Table 1. Photophysical data of investigated Pt (II) complexes with salen ligands.

\begin{tabular}{|c|c|c|c|c|}
\hline \multirow{2}{*}{ Complex } & \multicolumn{3}{|c|}{$\lambda_{\text {abs }}(\mathrm{nm})\left(\varepsilon, \mathrm{M}^{-1} \mathrm{~cm}^{-1}\right)$} & \multirow{2}{*}{$\lambda_{\text {em }}\left(\lambda_{\text {exc }}\right)(\mathrm{nm})$} \\
\hline & $\pi-\pi^{*}$ & $n-\pi^{*}$ & MLCT & \\
\hline 1 & $317(20,415)$ & $342(24,840)$ & $422(9,200)$ & 549 (422) \\
\hline 2 & $292(11,045)$ & $350(5,055)$ & $429(6,720)$ & $572(429)$ \\
\hline 3 & $300(24,210)$ & $362(5,930)$ & $424(8,405)$ & $561(424)$ \\
\hline 4 & $305(29,615)$ & $368(9,975)$ & $398(6,960)$ & $525(398)$ \\
\hline 5 & $320(14,130)$ & $360(10,980)$ & $446(8,740)$ & $601(446)$ \\
\hline 6 & $325(19,725)$ & $358(15,050)$ & $410(5,585)$ & 545 (410) \\
\hline
\end{tabular}

\subsection{In vitro Cytotoxicity Assay}

The cytotoxicity of studied Pt (II) complexes was estimated by using MTT assay as described previously [21]. In brief, human epidermoid carcinoma cells (KB) or breast cancer cells (MCF-7) were cultured and maintained in DMEM which was added with 10\% FBS (Fetal Bovine
Serum) and $1 \%$ penicillin streptomycin at $37^{\circ} \mathrm{C}$ in humidified atmosphere with $5 \% \mathrm{CO}_{2}$ and $95 \%$ air. Testing samples of $\mathrm{Pt}$ (II) complexes were dissolved in DMSO to give various concentrations of $0-128 \mu \mathrm{g} / \mathrm{ml}$. Approximately 10,000 cancer cells were seeded into each well of a 96 well micro titer plate and incubated $24 \mathrm{~h}$. Then $10 \mu \mathrm{L}$ solution containing various 
concentrations of different Pt (II)-salen complexes and 190 $\mu \mathrm{L}$ DMEM solution containing cancer cells were added into each wells and incubated for additional $72 \mathrm{~h}$ under standard growing condition. The cell viability was analyzed by using MTT assay based on the absorbance of the lysates at $540 \mathrm{~nm}$ on Genios TECAN spectrophotometter. The percent viable cells were plotted as a function of concentration to determine the $\mathrm{IC}_{50}$ values. The each experiment was carried out triplicate. The obtained $\mathrm{IC}_{50}$ values of studied complexes were presented in Table 2 .
Table 2. The in vitro cytotoxcity of Pt (II) complexes with salen ligands.

\begin{tabular}{lll}
\hline \multirow{2}{*}{ Complex } & $\mathbf{I C}_{\mathbf{5 0}}(\boldsymbol{\mu M})$ & \\
\cline { 2 - 3 } & $\mathbf{K B}$ & $\mathbf{M C F}-\mathbf{7}$ \\
\hline 1 & $3.15[20]$ & $13.45[20]$ \\
2 & 8.05 & 8.05 \\
3 & 5.37 & 7.68 \\
4 & 1.92 & 1.92 \\
5 & 2.88 & 3.26 \\
6 & 7.68 & 25.53 \\
Ellipticine & 1.14 & 2.03 \\
\hline
\end{tabular}

\section{Discussion}

\subsection{Synthesis and Characterization}

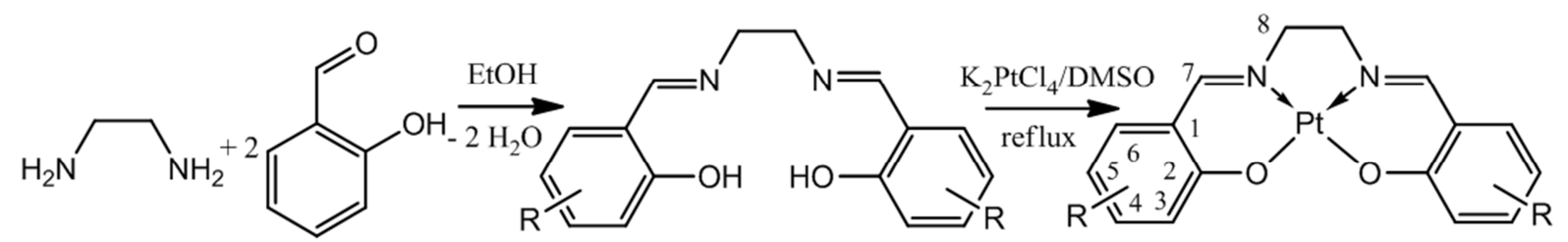

Figure 1. Synthesis of salen ligands and complexes.

Table 3. Salen ligands and their Platinum (II) complexes.

\begin{tabular}{|c|c|c|}
\hline $\mathbf{R}$ & Ligand & Complex \\
\hline $\mathrm{H}$ & Salen $_{2}$ & [Pt (II) (salen)] (1) \\
\hline $5-\mathrm{F}$ & 5-FsalenH2 & [Pt (II) (5-Fsalen)] (2) \\
\hline 3-MeO & 3-MeOsalenH $\mathrm{H}_{2}$ & [Pt (II) (3-MeOsalen)] (3) \\
\hline 4-MeO & 4-MeOsalenH $\mathrm{H}_{2}$ & [Pt (II) (4-MeOsalen)] (4) \\
\hline $5-\mathrm{MeO}$ & 5-MeOsalenH $\mathrm{H}_{2}$ & [Pt (II) (5-MeOsalen)] (5) \\
\hline 6-MeO & 6-MeOsalenH $\mathrm{H}_{2}$ & [Pt (II) (6-MeOsalen)] (6) \\
\hline
\end{tabular}

Firstly, the N, N'-bis (salicylidene)-1, 2-ethylenediimine ligands were synthesized in high yields (91-95\%). The commercial 1, 2-ethylenediamine was reacted with two equivalents of salicylaldehydes in ethanol as solvent at room temperature. The productive precipitates were collected and washed by cold ethanol to afford yellowish solids. Their ESIMS spectra received were in good agreement with their suggested formula weights. The bands at about 2532-2619 $\mathrm{cm}^{-1}$ in the IR spectra of ligands can be assigned to the $\mathrm{O}-\mathrm{H}$ stretching frequencies. The vibration band of $\mathrm{C}=\mathrm{N}$ was appeared in the frequencies of $1577-1639 \mathrm{~cm}^{-1}$. The characteristic bands found at about 1261-1296 and 1159$1250 \mathrm{~cm}^{-1}$ can belong to $\mathrm{C}-\mathrm{N}$ and $\mathrm{C}-\mathrm{O}$ stretching vibrations respectively (Table 4). In ${ }^{1} \mathrm{H}-\mathrm{NMR}$ spectra recorded in $\mathrm{CDCl}_{3}$, the typical signals at about $12.68-14.18 \mathrm{ppm}$ can be assigned to the $\mathrm{OH}$ proton peaks. The signals at $8.20-8.80$ ppm can assure the ligand formulation bonding $\mathrm{CH}=\mathrm{N}$. The proton peaks of ethylene were found at 3.85-3.96 ppm. The substituted groups at salicylidene moiety afforded the characteristic signals in ${ }^{1} \mathrm{H}-\mathrm{NMR}$. The proton signals of salicyl ring with fluoro at 5-position observed at 6.89-7.02 ppm were in multilets. The proton signals of $\mathrm{MeO}$ groups of MeOsalen $\mathrm{H}_{2}$ ligands were appeared as a singlet in the field of 3.75-3.88 ppm (Table 5). ${ }^{13} \mathrm{C}-\mathrm{NMR}$ spectra showed the characteristic peaks at about $156.00-166.68$ ppm for $\mathrm{C}-\mathrm{O}$, 151.49-163.53 ppm for $\mathrm{C}=\mathrm{N}$ and 58.85-59.85 ppm for $\mathrm{CH}_{2} \mathrm{CH}_{2}$ carbon signals. All carbon peaks of salicyl ring with fluoro at 5-positon were appeared in double ones. The carbon signals of $\mathrm{MeO}$ groups of $\mathrm{MeOsalenH}_{2}$ ligands were at about 55.34-56.11 ppm (Table 6).

Then a series of Pt (II) complexes were prepared in moderate yields $(65.5-68.5 \%)$ between synthetic salen ligands and $\mathrm{K}_{2} \mathrm{PtCl}_{4}$ in mild condition [Figure 1]. Their ESIMS spectra were suitable to their proposed molecular formulations (Table 3). In IR spectra, the characteristic peaks of ligands were found at $1600-1621 \mathrm{~cm}^{-1}$ for $\mathrm{C}=\mathrm{N}, 1192$ $1254 \mathrm{~cm}^{-1}$ for $\mathrm{C}-\mathrm{N}$ and $1106-1248 \mathrm{~cm}^{-1}$ for $\mathrm{C}-\mathrm{O}$ stretching vibrations. The vibrations of these bongdings were shifted to higher fields in coordination. New vibrations of $\mathrm{Pt}-\mathrm{N}$ and $\mathrm{Pt}-$ $\mathrm{O}$ for the coordination between Pt (II) and tetradentate salen ligands were observed at 611-631 and 417-434 $\mathrm{cm}^{-1}$ respectively (Table 4).

Table 4. Main IR absorption of ligands and Pt (II) complexes $\left(\mathrm{cm}^{-1}\right)$.

\begin{tabular}{|c|c|c|c|c|c|c|}
\hline Compound & $v_{(O-H)}\left(\mathrm{cm}^{-1}\right)$ & $v_{(C=N)}\left(\mathrm{cm}^{-1}\right)$ & $v_{(C-N)}\left(\mathrm{cm}^{-1}\right)$ & $v_{(C-O)}\left(\mathrm{cm}^{-1}\right)$ & $v_{(\mathrm{Pt}-\mathrm{N})}\left(\mathrm{cm}^{-1}\right)$ & $v_{(\mathrm{Pt}-\mathrm{O})}\left(\mathrm{cm}^{-1}\right)$ \\
\hline SalenH $_{2}$ & 2549 & 1636 & 1283 & 1199 & - & - \\
\hline 5-FsalenH2 & 2571 & 1634 & 1272 & 1225 & - & - \\
\hline 3-MeOsalenH ${ }_{2}$ & 2555 & 1633 & 1296 & 1250 & - & - \\
\hline
\end{tabular}




\begin{tabular}{|c|c|c|c|c|c|c|}
\hline Compound & $\mathrm{v}_{(\mathrm{O}-\mathrm{H})}\left(\mathrm{cm}^{-1}\right)$ & $\mathrm{V}_{(\mathrm{C}=\mathrm{N})}\left(\mathrm{cm}^{-1}\right)$ & $v_{(\mathrm{C}-\mathrm{N})}\left(\mathrm{cm}^{-1}\right)$ & $v_{(\mathrm{C}-O)}\left(\mathrm{cm}^{-1}\right)$ & $v_{(\mathrm{Pt}-\mathrm{N})}\left(\mathrm{cm}^{-1}\right)$ & $\mathrm{v}_{(\mathrm{Pt}-\mathrm{O})}\left(\mathrm{cm}^{-1}\right)$ \\
\hline 4-MeOsalenH $\mathrm{H}_{2}$ & 2551 & 1620 & 1285 & 1223 & - & - \\
\hline 5-MeOsalenH $\mathrm{H}_{2}$ & 2571 & 1639 & 1276 & 1159 & - & - \\
\hline 6-MeOsalenH $\mathrm{H}_{2}$ & 2531 & 1627 & 1295 & 1250 & - & - \\
\hline 1 & - & 1621 & 1192 & 1127 & 611 & 417 \\
\hline 2 & - & 1612 & 1221 & 1127 & 618 & 434 \\
\hline 3 & - & 1604 & 1247 & 1126 & 631 & 433 \\
\hline 4 & - & 1600 & 1228 & 1124 & 625 & 432 \\
\hline 5 & - & 1607 & 1220 & 1148 & 613 & 427 \\
\hline 6 & - & 1607 & 1254 & 1106 & 623 & 434 \\
\hline
\end{tabular}

In ${ }^{1} \mathrm{H}$-NMR spectra of $\mathrm{Pt}$ (II) complexes recorded in DMSO- $d_{6}$, there were no signal for $\mathrm{OH}$ groups in low field showing that the separation of $\mathrm{H}$ in coordination. Other typical proton signals were found in a singlet at $8.02-8.77 \mathrm{ppm}$ for $\mathrm{CH}=\mathrm{N}$ and a singlet at 3.80-4.46 ppm for $\mathrm{CH}_{2} \mathrm{CH}_{2}$ groups. The protons of complex 2 salicyl ring with fluoro at 5-position was observed in multilets. The proton peaks of $\mathrm{MeO}$ group in [Pt (II) MeOsalen] complexes were appeared as a singlet at 3.69-3.82 ppm (Table $5)$.

Table 5. Typical ${ }^{1} H-N M R$ signals of salen ligands and Pt (II) complexes (ppm).

\begin{tabular}{|c|c|c|c|c|}
\hline Compound & $\delta_{(\mathrm{OH})}(\mathrm{ppm})$ & $\delta_{(\mathrm{CH}=\mathrm{N})}(\mathrm{ppm})$ & $\delta_{(\mathrm{CH} 2 \mathrm{CH} 2)}(\mathrm{ppm})$ & $\delta_{(\mathrm{MeO})}(\mathrm{ppm})$ \\
\hline SalenH $_{2}$ & 13.18 & 8.35 & 3.93 & - \\
\hline 5-FsalenH2 & 12.85 & 8.30 & 3.96 & - \\
\hline 3-MeOsalenH ${ }_{2}$ & 13.55 & 8.32 & 3.95 & 3.88 \\
\hline 5-MeOsalenH $\mathrm{H}_{2}$ & 12.68 & 8.30 & 3.94 & 3.75 \\
\hline 6-MeOsalenH $\mathrm{H}_{2}$ & 14.18 & 8.80 & 3.88 & 3.78 \\
\hline 1 & - & 8.54 & 3.82 & - \\
\hline 3 & - & 8.52 & 3.81 & 3.77 \\
\hline 4 & - & 8.02 & 4.32 & 3.73 \\
\hline 5 & - & 8.47 & 3.80 & 3.69 \\
\hline 6 & - & 8.77 & 3.82 & 3.82 \\
\hline
\end{tabular}

In ${ }^{13} \mathrm{C}-\mathrm{NMR}$ spectra, the typical carbon signals presentating for $\mathrm{C}-\mathrm{O}$ were found at $156.00-163.21 \mathrm{ppm}, \mathrm{C}=\mathrm{N}$ at 153.53 161.81 and $\mathrm{CH}_{2} \mathrm{CH}_{2}$ at $60.49-61.58 \mathrm{ppm}$. Other carbon signals of salicylidene moiety with fluoro at 5-position in complex 2 were observed as double peaks at about $117.00-153.83 \mathrm{ppm}$. The carbon signals of $\mathrm{MeO}$ groups in [Pt (II)(MeOsalen)] complexes were appeared at 55.18-55.84 ppm (Table 6).

Table 6. Typical ${ }^{13} \mathrm{C}-\mathrm{NMR}$ signals of salen ligands and Pt (II) complexes (ppm).

\begin{tabular}{|c|c|c|c|c|}
\hline Compound & $\delta_{(\mathrm{OH})}(\mathrm{ppm})$ & $\delta_{(\mathrm{CH}=\mathrm{N})}(\mathbf{p p m})$ & $\delta_{(\mathrm{CH} 2 \mathrm{CH} 2)}(\mathrm{ppm})$ & $\delta_{(\mathrm{MeO})}(\mathrm{ppm})$ \\
\hline $\mathrm{SalenH}_{2}$ & 166.51 & 161.03 & 59.75 & - \\
\hline 5-FsalenH2 & 165.58 and 165.56 & 154.60 & 59.71 & - \\
\hline 3-MeOsalenH ${ }_{2}$ & 166.68 & 151.49 & 59.50 & 56.11 \\
\hline 4-MeOsalenH ${ }_{2}$ & 165.43 & 163.53 & 58.85 & 55.34 \\
\hline 5-MeOsalenH $\mathrm{H}_{2}$ & 166.27 & 155.17 & 59.85 & 55.95 \\
\hline 6-MeOsalenH $\mathrm{H}_{2}$ & 163.80 & 162.63 & 59.65 & 55.57 \\
\hline 1 & 162.45 & 155.98 & 60.92 & - \\
\hline 2 & 162.62 & 157.98 & 60.49 & - \\
\hline 3 & 156.00 & 153.53 & 60.92 & 55.18 \\
\hline 4 & 163.13 & 161.81 & 60.53 & 55.39 \\
\hline 5 & 157.73 & 155.41 & 60.95 & 55.53 \\
\hline 6 & 163.21 & 159.36 & 61.58 & 55.84 \\
\hline
\end{tabular}

\subsection{Photophysical Properties}

The photophysical data of Pt (II) complexes in dilute DCM solutions were presented in Table 1.

When dissolved in DCM, Pt (II) complexes gave yellow solutions and exhibited UV-Visible absorption spectra with $\lambda_{\max }$ at about $292-446 \mathrm{~nm}$ of three bands. Two bands at $292-$ 325 and 342-368 nm could featured for high-energy intraligand absorption of $\pi \rightarrow \pi^{*}$ and $n \rightarrow \pi^{*}$ transitions respectively and a low-energy metal-to-ligand charge transfer (MLCT) absorption must be at $398-446 \mathrm{~nm}$ probably (Figure 2).
Compared to complex 1, both electron-withdrawing and donoring substituted groups at 5-position of salicyl rings of complex 2 and 5 respectively shifted MLCT absorption bands to longer wavelengths. Methoxy group at different position of salicyl rings also perform obvious impact to MLCT absorption wavelengths of investigated complexes. Methoxy group at 3 or 5 -position shifted wavelength to longer range while methoxy at 4 or 6-position moved the wavelength to shoter band (Table 1). 


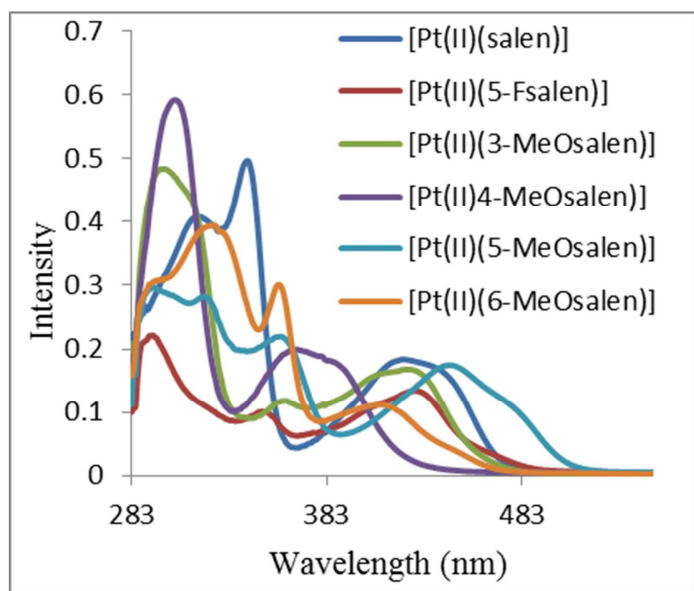

Figure 2. UV-Visible absorption spectra.

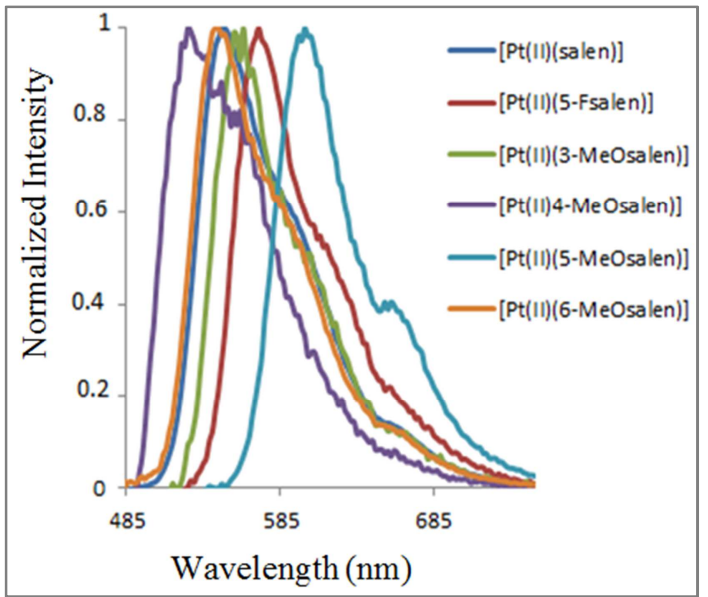

Figure 3. Emission spectra of Pt (II) complexes.

The emission spectra in solution of these complexes exhibited $\lambda_{\max }$ at about $525-601 \mathrm{~nm}$. The substituted group of fluoro at salicyl ring 5-position may shift emission spectra of complex 2 to red region. Methoxy group at different positions of salicyl ring could also affect to Pt (II) complexes' emission wavelengths. Methoxy group at 3- or 5-position of salicyl ring could shift emission band to red range and methoxy group at salicyl ring 4- or 6- postion must shift emission band to blue region (Figure 3).

\subsection{In Vitro Cytotoxicity}

In order to observe possible structure-activity relationships, the in vitro cytotoxicity of obtained platinum (II) complexes was evaluated against two human cancer cell lines of KB and MCF-7. The $\mathrm{IC}_{50}$ values (the concentration that inhibited in $50 \%$ the cellular proliferation) was presented in Table 2 . It was noted that all investigated platinum (II) complexes have antiproliferative effectivity with low $\mathrm{IC}_{50}$ values $(<50 \mu \mathrm{M})$. The effect of the substituted groups of studied ligands to complexes' cytotoxicity was quite obvious. In details, electron donoring methoxy at salicyl ring 5-position in complex 5 gave better activity than electron withdrawing fluoro in complex 2. The methoxy at different position of salicylidene moiety also showed the different effect on both human cancer cell lines. Methoxy at 4- or 5-position afforded the better results the one at 3- or 6-position. Compared to the standard anticancer compound, complex 4 gave the anticancer activity with $\mathrm{IC}_{50}=1.92 \mu \mathrm{M}$ for both $\mathrm{KB}$ and MCF-7 similar to the activity of standard ellipticine, $\mathrm{IC}_{50}=$ $1.14 \mu \mathrm{M}$ for $\mathrm{KB}$ and 2.03 for MCF-7. It can be seen that the in vitro cytotoxicity of this series of platinum (II) complexes increases in the sequence $2<6<3<1<5<4$ for BK and 6 $<1<2<3<5<4$ for MCF-7 cancer cell lines.

\section{Conclusions}

In this study, a series of platinum (II) complexes with salen ligands were synthesized and characterized by ESI-MS, IR and NMR spectroscopies. The photophysical properties estimated by UV-Visible absorption and emission spectra show that electron-withdrawing or -donoring substituted groups at 5-position of salicyl rings shifted MLCT absorption and emission bands to longer wavelengths. Methoxy group at different position of salicyl rings also perform obvious effect to MLCT absorption and emission wavelengths of investigated complexes. Methoxy group at 3 or 5-position shifted wavelength to longer range while methoxy at 4 or 6position moved the wavelength to shoter one. The biological activity of all complexes were examined against human cancer cell lines of $\mathrm{KB}$ and $\mathrm{MCF}-7$, the results showed that all complexes have influenced $\mathrm{KB}$ and $\mathrm{MCF}-7$ cell lines with low $\mathrm{IC}_{50}$ values $(<50 \mu \mathrm{M})$. Complex 4 gave the best activity for both $\mathrm{KB}$ and MCF-7 with $\mathrm{IC}_{50}=1.92 \mu \mathrm{M}$ which was similar to the cytotoxicity of standard anticancer compound, ellipticine, 1.14 and $2.03 \mu \mathrm{M}$ respectively.

\section{Acknowledgements}

This work is financially supported by Vietnam Academy of Science and Technology under grant number VAST04.01/17-18.

\section{References}

[1] N. Muhammad, Z. Guo. Metal-based anticancer chemotherapeutic agents. Cur. Op. Chem. Biol. 19 (2014) 144-153.

[2] U. Kalinowska-Lis, J. Ochocki, K. Matlawska-Wasowska. Trans geometry in platinum antitumor complexes. Coor. Chem. Rev. 252 (2008) 1328-1345.

[3] P. Heffeter, U. Jungwirth, M. Jakupec, C. Hartinger, M. Galanski, L. Elbling, M. Micksche, B. Keppler, W. Berger. Resistance against novel anticancer metal compounds: Differences and similarities. Drug Resis. Upd. 11 (2008) 1-16.

[4] Anne-Laure Laine, C. Passirani. Novel metal-based anticancer drugs: a new challenge in drug delivery. Cur. Op. Pharm. 12 (2012) 420-426.

[5] Pieter CA Bruijnincx, Peter J Sadler. New trends for metal complexes with anticancer activity. Cur. Op. Chem. Biol. 12 (2008) 197-206. 
[6] Cleiton M. da Silva, Daniel L. da Silva, Luzia V. Modolo, Rosemeire B. Alves, Maria A. de Resende, Cleide V. B. Martins, Ângelo de Fátima. Schiff bases: A short review of their antimicrobial activities. J. Adv. Research 2 (2011) 1-8.

[7] K. C. Gupta, A. K. Sutar. Catalytic activities of Schiff base transition metal complexes. Coor. Chem. Rev. 252 (2008) $1420-1450$

[8] A. Erxleben. Transition metal salen complexes in bioinorganic and medicinal chemistry. Inorg. Chim. Acta 472 (2018) 40-57.

[9] Y. Zhang, F. Meng, C. You, S. Yang, W. Xiong, Y. Wang, S. $\mathrm{Su}$, W. Zhu, Achieving NIR emission for tetradentate platinum (II) salophen complexes by attaching dual donoraccepter frameworks in the heads of salophen, Dyes and Pigments 138 (2017) 100-106.

[10] Y. Zhang, Z. Yin, F. Meng, J. Yu, C. You, S. Yang, H. Tan, W. Zhu, S. Su, Tetradentate Pt (II) 3, 6-substitued salophen complexes: Synthesis and tuning emission from deep-red to near infrared by appending donoracceptor framework, Organic Electronics 50 (2017) 317-324.

[11] Hirohiko Houjou, Yuki Hoga, Yi-Lan Ma, Hiroto Achira, Isao Yoshikawa, Toshiki Mutai, Kazunari Matsumura, Dinuclear fused salen complexes of group-10 metals: Peculiarity of the crystal structure and near-infrared luminescence of a bis (Ptsalen) complex, Inorg. Chim. Acta 461 (2017) 27-34.

[12] H. Achira, Y. Hoga, I. Yoshikawa, T. Mutai, K. Matsumura, H. Houjou, Effects of a semiflexible linker on the mechanochromic photoluminescence of bis (Pt-salen) complex, Polyhedron 113 (2016) 123-131.

[13] J. Zhang, G. Dai, F. Wu, D. Li, D. Gao, H. Jin, S. Chen, X. Zhu, C. Huang, D. Han. Efficient and tunable phosphorescence of new platinum (II) complexes based on the donor- $\pi$-acceptor Schiff bases. J. Photochem. Photobiol A: Chem. 316 (2016) 12-18.

[14] M. Hashemi, Z. Solati, A. Ghodsi, S. Ahmadian. Azo- substituted Schiff base complex of Pt (II): Synthesis, characterization, DFT and TD-DFT study. Syn. Metals 210 (2015) 398-403.

[15] Peng Wu, Dik-Lung Ma, Chung-Hang Leung, Siu-Cheong Yan, Nianyong Zhu, R. Abagyan, Chi-Ming Che. Stabilization of G-quadruplex DNA with platinum (II) Schiff base complexes: Luminescent probe and down-regulation of c-myc oncogene expression. Chem. Eur. J. 15 (2009) 13008 -13021.

[16] M. Proetto, W. Liu, A. Hagenbach, U. Abram, R. Gust. Synthesis, characterization and in vitro antitumour activity of a series of novel platinum (II) complexes bearing Schiff base ligands. Eur. J. Med. Chem. 53 (2012) 168 - 175.

[17] L. Li, C. Tian, C. Wang, G. Wang, L. Wang, J. Du. Platinum (II) complexes with tetradentate Schiff bases as ligands: Synthesis, characterization and detection of DNA interaction by differential pulse voltammetry. E-Journal of Chemistry 9 (3) (2012) 1422-1430.

[18] M. Azam, S. I. Al-Resayes, S. M. Soliman, A. TrzesowskaKruszynska, R. Kruszynski, Z. Khan. A (salicyidiminato) Pt (II) complex with dimethylpropylene linkage: Synthesis, structural characterization and antineoplatic activity. J. Photochem. Photobiol. B: Biol. 176 (2017) 150-156.

[19] H. Naeimi, J. Safari, A. Heidarnezhad. Synthesis of Schiff base ligands derived from condensation of salicylaldehyde derivatives and synthetic diamine. Dyes and Pigments 73 (2007) 251-253.

[20] Quang Trung Nguyen, Quang Hai Lam, Van Tuyen Nguyen. Synthesis, characterization and in vitro antitumour activity of $\mathrm{Pt}$ (II) complexes of salen type Schiff base ligands. Vietnam J. Chem. 54 (6e2) (2016) 219-222.

[21] K. I. Ansari, J. D. Grant, S. Kasiri, G. Woldemariam, B. Shrestha, S. S. Mandal. Manganese (III)-salens induce tumor selective apoptosis in human cells. J. Inorg. Biochem. 103 (2009) 818-826. 